\title{
Oxides of small Rhodium clusters: Theoretical investigation of experimental reactivities
}

Cite as: J. Chem. Phys. 129, 124304 (2008); https://doi.org/10.1063/1.2981810

Submitted: 31 July 2008 . Accepted: 25 August 2008 . Published Online: 23 September 2008

D. J. Harding, R. D. L. Davies, S. R. Mackenzie, and T. R. Walsh

\section{ARTICLES YOU MAY BE INTERESTED IN}

Probing the structures of gas-phase rhodium cluster cations by far-infrared spectroscopy

The Journal of Chemical Physics 133, 214304 (2010); https://doi.org/10.1063/1.3509778

Communications: The structure of $\mathrm{Rh}_{8}^{+}$in the gas phase

The Journal of Chemical Physics 132, 011101 (2010); https://doi.org/10.1063/1.3285266

C-H activation of alkanes on $\mathrm{Rh}_{n}^{+}(n=1-30)$ clusters: Size effects on dehydrogenation

The Journal of Chemical Physics 123, 214709 (2005); https://doi.org/10.1063/1.2131066

The Journal

Submit Today

of Chemical Physics

The Emerging Investigators Special Collection and Awards Recognizing the excellent work of early career researchers! 


\title{
Oxides of small Rhodium clusters: Theoretical investigation of experimental reactivities
}

\author{
D. J. Harding, ${ }^{1}$ R. D. L. Davies, ${ }^{1}$ S. R. Mackenzie, ${ }^{3}$ and T. R. Walsh ${ }^{1,2, a)}$ \\ ${ }^{1}$ Department of Chemistry, University of Warwick, Coventry CV4 7AL, United Kingdom \\ ${ }^{2}$ Centre for Scientific Computing, University of Warwick, Coventry CV4 7AL, United Kingdom \\ ${ }^{3}$ University Chemical Laboratories, Lensfield Road, Cambridge CB2 1EW, United Kingdom
}

(Received 31 July 2008; accepted 25 August 2008; published online 23 September 2008)

\begin{abstract}
Density functional theory is used to investigate the structures of cationic rhodium cluster oxides, $\mathrm{Rh}_{6} \mathrm{O}_{m}^{+}(m=1,4)$. On the monoxide and dioxide, the oxygen atoms occupy bridge sites, while on trioxide and tetroxide clusters, high-coordination sites are favored. A range of spin multiplicities are investigated for each cluster, with high spin multiplicities found to be less favored for the oxides compared with the naked metal clusters. The dissociation of nitric oxide on low-energy isomers of $\mathrm{Rh}_{6} \mathrm{O}_{4}^{+}$is investigated and found to be unfavorable compared to molecular adsorption due to a combination of thermodynamic and kinetic factors. These calculations are consistent with, and help to account for, the experimentally observed reactivity of rhodium and rhodium oxide clusters with nitric oxide [M. S. Ford et al., Phys. Chem. Chem. Phys. 7, 975 (2005)]. () 2008 American Institute of Physics. [DOI: 10.1063/1.2981810]
\end{abstract}

\section{INTRODUCTION}

The properties of transition-metal (TM) clusters have been observed to exhibit large variations with cluster size, ${ }^{1}$ often differing with both atomic and bulk phases. Stark examples in literature include cluster ionization potentials, ${ }^{2-5}$ magnetic moments, ${ }^{6-8}$ and reactivities toward small molecules. ${ }^{9-11}$ Cluster structure has been suggested to play a significant role, as elucidated by both theory and experiment. In particular, the size range over which properties show the greatest variation is up to around 20 atoms. Quantum chemical approaches are particularly amenable to the small system sizes inherent to this problem and have been shown to be a useful tool e.g., in assignment of cluster structures via vibrational spectra ${ }^{12}$ and understanding cluster reactivity. ${ }^{13}$

Gas-phase TM clusters have been proposed as model systems for the TM-based heterogeneous catalysts, ${ }^{1,14}$ e.g., in car catalytic converters, where rhodium is often used to remove nitrogen oxides from exhaust gases. ${ }^{15}$ The reactions of charged Rh clusters with nitric oxide (NO) have previously been studied by Fourier transform ion cyclotron resonance mass spectrometry, ${ }^{10,16}$ where small clusters were found to react with multiple NO molecules via the stepwise reaction,

$$
\begin{aligned}
& \mathrm{Rh}_{n} \mathrm{O}_{m}^{+}+\mathrm{NO} \rightarrow \mathrm{Rh}_{n} \mathrm{O}_{m} \mathrm{NO}^{+}, \\
& \mathrm{Rh}_{n} \mathrm{O}_{m} \mathrm{NO}^{+}+\mathrm{NO} \rightarrow \mathrm{Rh}_{n} \mathrm{O}_{m+2}^{+}+\mathrm{N}_{2},
\end{aligned}
$$

where $m=0,2,4$, etc. The mechanism is thought to proceed via the dissociative adsorption of $\mathrm{NO}$ on the cluster surface. The elementary step that liberates $\mathrm{N}_{2}$ is observed to occur until a certain size-dependent number of oxygen atoms are adsorbed on the cluster, after which point there is no more evidence to suggest dissociation of further $\mathrm{NO}$ molecules. For $\mathrm{Rh}_{6}^{+}$this reaction end point is observed at $\mathrm{Rh}_{6} \mathrm{O}_{4}^{+}$. These

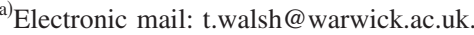

data suggest that the adsorbed oxygen atoms are able to block or change the sites which mediate dissociation of NO on the cluster. Three general situations for this end point can be envisaged, none of which can be resolved with mass spectrometry: (1) dissociation of neither the fifth nor the sixth NO molecules is favorable, (2) the fifth NO can dissociate but the sixth NO cannot, or finally, (3) both NO molecules can dissociate, but the nitrogen atoms are unable to recombine on the surface possibly due to large energy penalties for migration on the oxide cluster. For cases 1 and 2, this change in reactivity as oxygen atoms are adsorbed has parallels with the poisoning of catalysts.

The structures and binding of adsorbates on TM clusters have been investigated using both experimental and computational methods. The preferred binding sites of oxygen and hydrogen atoms on small neutral $\mathrm{Rh}_{n}$ clusters $(1 \leq n \leq 6)$ have been investigated by Mainardi and Balbuena ${ }^{17}$ using density-functional theory (DFT). For oxygen atoms, bridge binding sites were found to be favored for most of the cluster sizes investigated. Fielicke and co-workers ${ }^{18,19}$ used infrared (IR) multiphoton dissociation spectroscopy and DFT to probe the binding of $\mathrm{CO}$ on neutral and charged clusters of rhodium and found atop binding sites to be favored on most rhodium clusters, although two- and three-coordinated binding modes were also identified for some cluster sizes. The adsorption and reaction of $\mathrm{NO}$ on other TM clusters have also been explored. ${ }^{20,21}$

Comparison with extended surfaces is also useful. Oxygen atoms adsorbed on Rh single-crystal surfaces favor highcoordination hollow sites, e.g., the three-coordinate hollow site on $\mathrm{Rh}(111)$ (Ref. 22) and the four-coordinated hollow site on $\mathrm{Rh}(100){ }^{23}$ Computational and experimental studies $^{24-27}$ indicate that oxygen adsorption can induce surface reconstruction. The reactions of NO on rhodium surfaces have been widely studied, with significant differences 
in reactivity found between the different surfaces and sites. $^{28,29}$ Borg et $_{\text {al. }}{ }^{30}$ reported that the dissociation of $\mathrm{NO}$ on $\mathrm{Rh}(111)$ is hindered at high coverage, suggesting that several empty, adjacent, three-coordinated hollow sites are necessary for the dissociation of NO under these conditions.

The structures of the bare $\mathrm{Rh}_{6}^{+}$cluster have previously been investigated ${ }^{13}$ in an attempt to rationalize the biexponential reaction kinetics observed experimentally. ${ }^{16}$ These structures provide a platform from which to investigate the structures of the oxides of $\mathrm{Rh}_{6}^{+}$. We argue that the sequential heating of the clusters (as NO molecules dissociatively adsorb) and cooling (as $\mathrm{N}_{2}$ desorbs) during the reaction may be expected to effectively anneal the clusters, allowing the system to explore high energy regions of the potential energy surface (PES) before desorption of $\mathrm{N}_{2}$ quenches the system into relatively low-energy structures. Preliminary DFT calculations predict binding energies of approximately $2.8 \mathrm{eV}$ per dissociated NO molecule and approximately $0.8 \mathrm{eV}$ per molecularly adsorbed $\mathrm{N}_{2}$. As it is currently impractical to perform all possible pathway calculations for each step of the reaction, structures of the oxides were built up in a sequential manner from monoxide to tetroxide, to try to account for any structural rearrangement which may occur, ${ }^{31}$ in order to locate the candidate low-energy structure(s) of $\mathrm{Rh}_{6} \mathrm{O}_{4}^{+}$. However, in general, one cannot be certain of having found the global minimum or even all of the possible low-energy structures. In our system, the binding sites of the oxygen atom in the $\mathrm{Rh}_{6} \mathrm{O}^{+}$cluster are sufficiently small in number to be enumerated exhaustively; something that is unlikely to be possible for larger clusters. Therefore, we anticipate that the results presented herein will serve as useful benchmarks for future global optimization studies that employ novel empirical or semiempirical potentials.

In this work, we have used DFT calculations to investigate the structures and energetics of $\mathrm{Rh}_{6} \mathrm{O}_{m}^{+}, m=1-4$, and the dissociation of $\mathrm{NO}$ on low-energy structures of $\mathrm{Rh}_{6} \mathrm{O}_{4}^{+}$. We have used these data to explore the possible reaction endpoint scenarios outlined above, to determine which, if any, may be responsible for the experimentally observed changes in reactivity as oxygen atoms are added to rhodium cluster cations.

\section{METHODS}

Unlike our previous studies of naked TM clusters, ${ }^{13,32}$ the combination of rhodium and oxygen atoms prevents the use of empirical potentials in our search of the PES for candidate structures. Initial structures of $\mathrm{Rh}_{6} \mathrm{O}_{m}^{+}$were created by addition of an oxygen atom to low-energy structures of $\mathrm{Rh}_{6} \mathrm{O}_{m-1}^{+}$. These structures were used as input for DFT geometry optimization using the GAUSSIAN 03 package. ${ }^{33}$ The octahedron and prism geometries of the bare metal cluster were used as seed structures; in the octet state the bare prism is $0.32 \mathrm{eV}$ higher in energy than the octahedron. ${ }^{13}$ All calculations were performed using the Perdew-Burke-Ernzerhof (PBE) exchange-correlation functional. ${ }^{34}$ The Stuttgart/ Dresden (SDD) (Ref. 35) effective-core potential and valence Gaussian basis functions were used for the rhodium atoms and aug-cc-pVDZ basis functions ${ }^{36}$ were used for oxy- gen and nitrogen. A range of spin multiplicities from doublet to dectet were considered. Analytic calculation of the vibrational frequencies showed the minimized structures to be true minima at this level of theory; similarly, transition states were shown to support one imaginary vibrational frequency.

A set of cluster isomers was identified for each of the oxides (from monoxide to tetroxide). The equilibrium population distribution of each set was then predicted using the harmonic superposition approximation ${ }^{37}$ in the microcanonical ensemble. The probability $P_{i}^{\text {eq }}$ of population of each accessible isomer $i$ at a total energy $E$ is given by

$$
P_{i}^{\mathrm{eq}}=\frac{\Omega_{i}(E)}{\Omega(E)},
$$

where $\Omega_{i}(E)$ is the vibrational density of states of isomer $i$, and $\Omega(E)$ is the total energy density of states, the sum of the vibrational density of states over all accessible isomers. $\Omega_{i}(E)$ can be calculated using

$$
\Omega_{i}(E)=\frac{2 N !}{h_{i}^{\mathrm{pg}}} \frac{\left(E-E_{i}\right)^{(\kappa-1)}}{\Gamma(\kappa) \Pi_{j=1}^{\kappa} \nu_{j}},
$$

where $N$ is the number of atoms, $h_{i}^{\mathrm{pg}}$ is the order of the point group of isomer $i, \kappa$ is the number of vibrational degrees of freedom, $\nu_{j}$ are the vibrational frequencies of isomer $i$, and $E_{i}$ is the relative energy of isomer $i$. Using the calculated structures, energies, and frequencies of each of the unique cluster isomers, the equilibrium population can be calculated at a range of different total energies.

The nomenclature used herein describes the clusters by underlying metal framework; $O$ for octahedral, $P$ for prism, $C$ for capped square pyramid (csp), $B$ for boat (capped trigonal bipyramid), and $E$ for edge-capped trigonal bipyramid. The number and binding site(s) of the oxygen atoms are shown by subscripts; $h$ for hollow sites, $b$ for bridge, and $a$ for atop; on the prism isomer, $t$ and $s$ denote binding on the trigonal and square sites, respectively. Where several isomers have the same number of oxygen binding sites they also have been given an index number.

\section{RESULTS AND DISCUSSION}

\section{A. Monoxide structures}

Three different modes of binding for the oxygen atom were considered; atop, bridge, and hollow sites, on the lowenergy octahedron and prism isomers. The optimized structures and relative energies of the different $\mathrm{Rh}_{6} \mathrm{O}^{+}$clusters are shown in Fig. 1 and Table I. The favored structure is found to be the ${ }^{8} O_{b}$, with a gap of $0.09 \mathrm{eV}$ to the next highest structure, the ${ }^{4} O_{a}$ geometry. There are six almost isoenergetic bridge and atop spin/structure isomers that lie around $0.2 \mathrm{eV}$ higher in energy. The structures with the oxygen bound at a hollow site are the least energetically favored; oxygen in the four-coordinated hollow site of the prism is found to move toward a corner, becoming essentially three coordinated. Input structures with the oxygen in the three-coordinated hollow site on the octahedral cluster were found to collapse to the bridge structure. The preference of the oxygen to bind at a bridge site has been reported by Mainardi and Balbuena ${ }^{17}$ for neutral clusters, in contrast to rhodium surfaces where a 

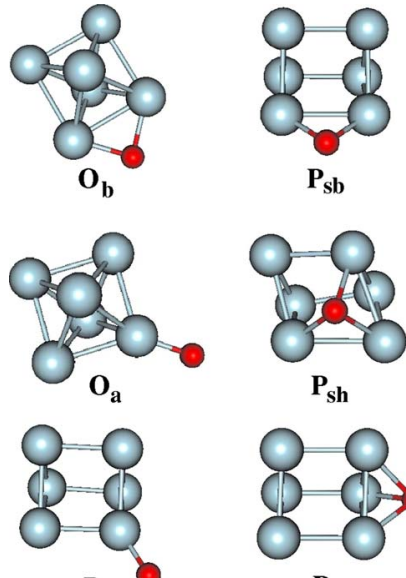

$\mathbf{P}_{\mathbf{a}}$

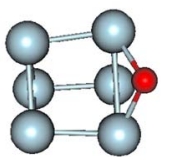

$\mathbf{P}_{\mathbf{t b}}$

FIG. 1. (Color online) The structures of $\mathrm{Rh}_{6} \mathrm{O}^{+}$clusters optimized at the PBE/SDD+aug-cc-pVDZ level of theory. Labels refer to those used in Table I.

hollow site is preferred. ${ }^{22,23}$ The binding of an oxygen on the surface of the cluster does not, in most cases, significantly distort the geometry of the underlying metal framework. However, binding to the four-coordinate hollow site on the square face of the prism structure has been found to cause slight distortion of the underlying metal structure toward the octahedral geometry. This distortion has some similarities with reconstruction observed following oxygen adsorption on the $\mathrm{Rh}$ (110) surface ${ }^{24-27}$ where oxygen binds in a threecoordinate site following reconstruction. The preference of the oxygen atom to bind at a bridge site suggested that input structures of higher oxides could be made by the addition of oxygen atoms at bridge sites.

\section{B. Di- and trioxide structures}

As building blocks toward the tetroxide structures of interest, and because they are formed in the reaction of $\mathrm{Rh}_{6}^{+}$ clusters with successive addition of molecules of $\mathrm{N}_{2} \mathrm{O},{ }^{11}$ the structures of di- and trioxides of $\mathrm{Rh}_{6}^{+}$clusters have been briefly investigated. Input structures for the dioxide have

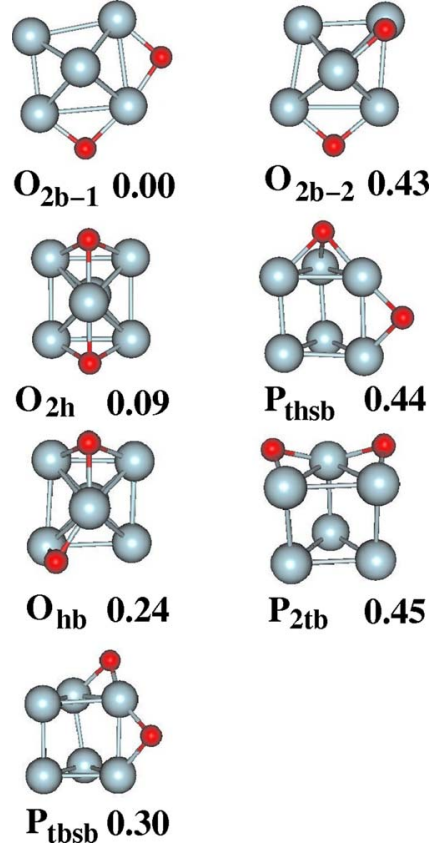

FIG. 2. (Color online) Low-energy structures and relative energies of ${ }^{8} \mathrm{Rh}_{6} \mathrm{O}_{2}^{+}$clusters. Energies are given in eV. Explanation of the labeling nomenclature is given in the text.

been made by adding an oxygen atom to all of the unique sites on the low-energy structures of the oxide. These structures were optimized in the octet spin state only to roughly map out the low-energy structures from which to build the trioxide input structures. The low-energy structures and relative energies identified for the dioxides in the octet state are shown in Fig. 2. The $O_{2 h}$ structure was formed by the collapse of a prism-based input structure to an octahedral based structure. The prism-based structures are higher in energy than octahedron-based structures. It was also found that some bridge oxygen atoms collapsed to hollow sites, though these resultant structures lie higher in energy than many of the corresponding bridge sites. Although we have not searched spin-multiplicity space thoroughly for the dioxides, the only conceivable doubt about spin effects concerns the ordering of the first two structures since the other structures are too high in energy. Our objective here was to use these data as a springboard for finding tetroxide structures not for obtaining the definitive global minimum for the dioxide in its own right.

TABLE I. Relative energies (eV) of $\mathrm{Rh}_{6} \mathrm{O}^{+}$clusters based on the prism and octahedron frameworks. The lowest energy spin multiplicity of each isomer is highlighted by bold text. Energies are calculated relative to the ${ }^{8} O_{b}$ structure.

\begin{tabular}{lcccccc}
\hline \hline & \multicolumn{7}{c}{ Spin multiplicity } \\
\cline { 2 - 7 } Structure & $\mathbf{2}$ & $\mathbf{4}$ & $\mathbf{6}$ & $\mathbf{8}$ & $\mathbf{1 0}$ & $\mathbf{1 2}$ \\
\hline$O_{b}$ & 0.20 & 0.32 & 0.18 & $\mathbf{0 . 0 0}$ & 0.28 & \\
$O_{a}$ & 0.13 & $\mathbf{0 . 0 9}$ & 0.11 & 0.21 & 0.22 & \\
$P_{a}$ & 0.20 & $\mathbf{0 . 1 7}$ & 0.37 & 0.57 & 0.88 & \\
$P_{\mathrm{tb}}$ & & 0.34 & $\mathbf{0 . 1 8}$ & 0.24 & 0.57 & \\
$P_{\mathrm{sb}}$ & 0.41 & $\mathbf{0 . 2 5}$ & 0.57 & 0.58 & 0.51 & 0.89 \\
$P_{\mathrm{sh}}$ & & 0.65 & $\mathbf{0 . 6 2}$ & 0.66 & 0.78 & \\
$P_{\mathrm{th}}$ & & 0.80 & $\mathbf{0 . 6 8}$ & 0.70 & 0.82 & \\
\hline \hline
\end{tabular}




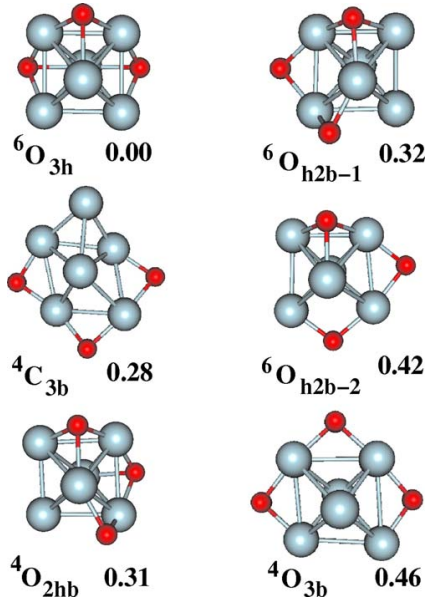

FIG. 3. (Color online) Structures and energies of the $\mathrm{Rh}_{6} \mathrm{O}_{3}^{+}$clusters, with relative energies given in $\mathrm{eV}$. For each distinct structure, we give the structure and energy for the spin multiplicity that yielded the lowest energy. Explanation of the labeling nomenclature is given in the text.

Guesses at trioxide structures were made based on a range of dioxide structures and optimized in a range of spin states from doublet to dectet $(22$ structures and 5 spin multiplicities). Figure 3 shows the structures and relative energies for the most favored geometries and spin multiplicities. It was found that different spin multiplicities of a small number of structures dominate (data not shown). Most of these low-energy structures are based on cappings of the octahedral $\mathrm{Rh}_{6}^{+}$framework, but the ${ }^{4} C_{3 b}$ structure is based on a capped square pyramid that collapsed from an optimization of an octahedron-based input structure. This and the rearrangement of one of the prism-based dioxide clusters show that oxygen adsorption can drive rearrangement of the underlying metal framework in these clusters. Oxygen adsorption on the threefold hollow site, which was found to be unfavorable on the octahedral based monoxide cluster, is found to be more favorable on the trioxide cluster, in common with bulk rhodium surfaces.

\section{Tetroxide structures and population distribution}

Tetroxide structures were based on cappings of the five trioxide geometries of lowest energy, and were optimized in spin multiplicities from doublet to dectet. There are 47 unique structures identified across the quartet to octet spin multiplicities within an energetic range of $1.5 \mathrm{eV}$ since some input structures collapsed to the same minimum upon optimization. The dectet and doublet structures are high in energy for all structures considered. The three structures found to dominate the population (vide infra) are shown in Fig. 4(a). Table II lists the relative energies of all spin/geometry combinations in a range up to $1.0 \mathrm{eV}$ greater than our putative global minimum. Again, it was found that different spin multiplicities for a small number of structures dominate in this energy range.

The population distribution of the $\mathrm{Rh}_{6} \mathrm{O}_{4}^{+}$isomers, as calculated using the harmonic superposition approximation, is shown in Fig. 4(b). It can be seen that at very low total energy the global minimum ${ }^{6} O_{4 h}$ structure dominates as all the other structures are too high in energy to be populated. In a)

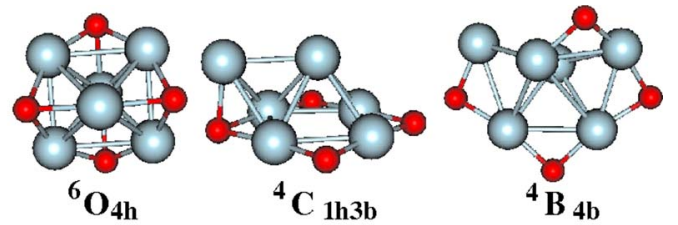

b)

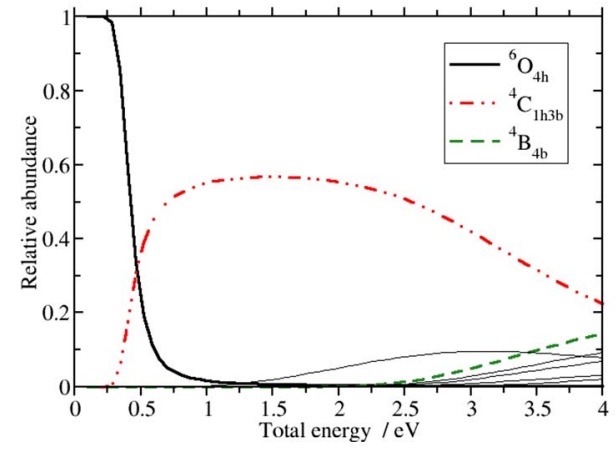

FIG. 4. (Color online) (a) The three geometric structures of $\mathrm{Rh}_{6} \mathrm{O}_{4}^{+}$that are predicted to be significantly abundant. Labels correspond with those given in Table II. (b) Structural abundance of $\mathrm{Rh}_{6} \mathrm{O}_{4}^{+}$as a function of total energy. All unique spin/geometry isomers were included in the calculation but only those with significant population are indicated.

the range from 0.5 to $3.5 \mathrm{eV}$ the ${ }^{4} C_{1 h 3 b}$ dominates the population. The rapid disappearance of the ${ }^{6} O_{4 h}$ and dominance of ${ }^{4} C_{1 h 3 b}$ at moderate energy can be attributed to the differences in the symmetry factors and vibrational frequencies of the two clusters; both factors appear in the denominator of Eq. (2), favoring structures of low symmetry or with low vibrational frequencies if all other factors are equal. The tetrahedral global minimum, ${ }^{6} \mathrm{O}_{4 h}$, has both higher symmetry and higher vibrational frequencies than the ${ }^{4} C_{1 h 3 b}$ structure; hence, it rapidly decreases in population at intermediate en-

TABLE II. Relative energies $(\Delta E / \mathrm{eV})$ of isomers of $\mathrm{Rh}_{6} \mathrm{O}_{4}^{+}$. All minima up to $1.0 \mathrm{eV}$ away from the putative global minimum are included. The structure is signified by the underlying metal geometry, the number of hollow $(h)$ and bridge $(b)$ oxygen atoms, and an index number (where necessary).

\begin{tabular}{lc}
\hline \hline Structure & $\Delta E(\mathrm{eV})$ \\
\hline${ }^{6} O_{4 h}$ & 0.00 \\
${ }^{4} C_{1 h 3 b}$ & 0.10 \\
${ }^{4} O_{4 h}$ & 0.16 \\
${ }^{8} O_{4 h}$ & 0.18 \\
${ }^{4} O_{2 h 2 b-1}$ & 0.30 \\
${ }^{6} C_{1 h 3 b}$ & 0.31 \\
${ }^{6} O_{3 h 1 b}$ & 0.37 \\
${ }^{4} O_{3 h 1 b}$ & 0.39 \\
${ }^{4} O_{4 b}$ & 0.48 \\
${ }^{6} O_{4 b}$ & 0.53 \\
${ }^{8} O_{4 b}$ & 0.60 \\
${ }^{4} E_{4 b}$ & 0.64 \\
${ }^{6} O_{2 h 2 b-1}$ & 0.64 \\
${ }^{8} C_{1 h 3 b}$ & 0.70 \\
${ }^{8} P_{4 b s q}$ & 0.71 \\
${ }^{4} B_{4 b}$ & 0.77 \\
${ }^{6} E_{4 b}$ & 0.82 \\
${ }^{6} O_{2 h 2 b-2}$ & 0.85 \\
${ }^{8} O_{2 h 2 b-1}$ & 0.98 \\
\hline \hline
\end{tabular}




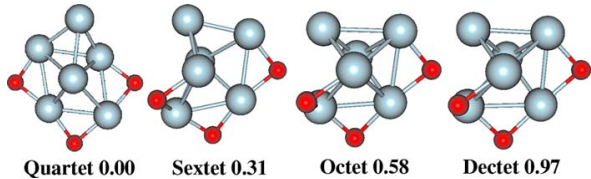

FIG. 5. (Color online) Structures and relative energies of $\mathrm{Rh}_{6} \mathrm{O}_{3}^{+}$clusters optimized from the same initial geometry $\left(O_{3 b}\right)$ but in different spin multiplicities. The spin multiplicity and relative energy $(\mathrm{eV})$ are shown.

ergies. At higher energy a number of structures with a range of spin multiplicities and geometries are present in the distribution with no single structure dominant. These structures have metal cores that resemble the higher energy isomers of the bare $\mathrm{Rh}_{6}^{+}$clusters such as csp and boat motifs. Previous calculations suggest the spin multiplicity to have less influence on the reactivity of TM clusters than the geometric structure. ${ }^{13}$ Therefore, the population of each unique cluster geometry can be calculated by summing over the populations of different spin multiplicities for each geometry. The equilibrium population distribution recalculated in this way exhibits very little difference with that shown in Fig. 4(b) and is not shown here.

\section{Effects of spin multiplicity on cluster structure}

For some oxide structures, the spin multiplicity appears to confer significant effects on both the relative energy of the structure and in some cases on the optimized structure itself. The favored spin multiplicities vary significantly with both the metal framework and the oxygen adsorption site, although quartets and sextets are generally favored for all the oxide structures compared to the octet and dectet states favored for most of the bare metal clusters. ${ }^{13}$ This observation is consistent with experimental ${ }^{7,8}$ and computational ${ }^{38-40}$ findings, which demonstrate that adsorption of a single atom or molecule can significantly alter the magnetic moment of some TM clusters.

Some isomers showed substantial structural dependence on spin state, as illustrated for the trioxide $O_{3 b}$ structure in Fig. 5, where optimization using the same input structure, but in different spin states, leads to resulting geometries with a different underlying metal framework. In this example, octet and dectet states led to roughly octahedral metal cores, while quartet and sextet states led to csp and boat geometries of the metal core. Both the boat and csp were previously identified as relatively low-energy isomers of $\mathrm{Rh}_{6}^{+13}$. This effect was not confined to this example alone but was observed for several other higher oxide structures. These data indicate that, at least for rhodium clusters, a range of spin multiplicities must be explored for each structure to gain a representative sample of low-energy configurations.

\section{E. Dissociation of $\mathrm{NO}$ on $\mathrm{Rh}_{6} \mathrm{O}_{4}^{+}$clusters}

As discussed in Sec. I, experimental results ${ }^{10,16}$ show a change in the reactivity of $\mathrm{Rh}_{6} \mathrm{O}_{n}^{+}$clusters around $n=4$, where dissociative adsorption of $\mathrm{NO}$ appears to give way to either molecular adsorption or trapping of adsorbed nitrogen atoms on the cluster surface. Transition states (TS) for the dissociation of $\mathrm{NO}$ on the two isomers of $\mathrm{Rh}_{6} \mathrm{O}_{4}^{+}$which are
TABLE III. Energetic data for reaction pathways on the tetroxide cluster. Total potential energy of reactant (a.u.), forward and reverse barriers $(\mathrm{eV})$ and difference in energy $\Delta E$ between reactant and product $(\mathrm{eV})$. With two exceptions, barriers were noted to not vary considerably with spin; therefore, for most cases we show indicative barriers for the quintet $O_{4 h}$ and triplet $C_{1 h 3 b}$ pathways.

\begin{tabular}{|c|c|c|c|c|}
\hline Pathway & Reactant (a.u.) & Forward (eV) & Reverse (eV) & $\Delta E_{\mathrm{rxn}}(\mathrm{eV})$ \\
\hline${ }^{5} \mathrm{O}_{4 h}-\mathrm{TS} 1$ & -1093.828309 & 2.43 & 1.19 & 1.24 \\
\hline${ }^{7} \mathrm{O}_{4 h}-\mathrm{TS} 1$ & -1093.799422 & 1.79 & 1.20 & 0.59 \\
\hline${ }^{5} \mathrm{O}_{4 h}$-TS2 & -1093.853354 & 3.57 & 1.28 & 2.30 \\
\hline${ }^{5} \mathrm{O}_{4 h}-\mathrm{TS} 3$ & -1093.863297 & 3.46 & 1.02 & 2.44 \\
\hline${ }^{5} \mathrm{O}_{4 h}$-TS4 & -1093.863251 & 4.99 & 2.42 & 2.57 \\
\hline${ }^{5} \mathrm{O}_{4 h}$-TS5 & -1093.847658 & 1.96 & 1.91 & 0.05 \\
\hline${ }^{7} \mathrm{O}_{4 h}$-TS5 & -1093.801751 & 0.76 & 0.98 & -0.22 \\
\hline${ }^{3} C_{1 h 3 b}-\mathrm{TS} 1$ & -1093.859790 & 1.96 & 0.38 & 1.58 \\
\hline${ }^{3} C_{1 h 3 b}-\mathrm{TS} 2$ & -1093.872488 & 2.05 & 0.43 & 1.62 \\
\hline${ }^{3} C_{1 h 3 b}-\mathrm{TS} 3$ & -1093.813890 & 1.10 & 2.08 & -0.97 \\
\hline${ }^{3} C_{1 h 3 b}-\mathrm{TS} 4$ & -1093.872487 & 2.35 & 0.63 & 1.72 \\
\hline${ }^{3} C_{1 h 3 b}$-TS5 & -1093.834179 & 1.95 & 0.34 & 1.62 \\
\hline${ }^{3} C_{1 h 3 b}$-TS6 & -1093.869011 & 2.71 & 0.30 & 2.42 \\
\hline${ }^{3} C_{1 h 3 b}$-TS7 & -1093.834932 & 0.64 & 0.93 & -0.28 \\
\hline${ }^{3} C_{1 h 3 b}$-TS 8 & -1093.870743 & 2.19 & 1.23 & 0.96 \\
\hline${ }^{3} C_{1 h 3 b}$-TS 9 & -1093.827799 & 2.60 & 0.32 & 2.28 \\
\hline
\end{tabular}

predicted to dominate the population at low $\left({ }^{6} \mathrm{O}_{4 h}\right)$ and intermediate $\left({ }^{4} C_{1 h 3 b}\right)$ energy have been identified. As NO is a radical, spin states of the cluster-NO complex with spin multiplicity one higher or one lower have been considered, i.e., quintets and septets for the ${ }^{6} \mathrm{O}_{4 h}$ based structure, and triplets and quintets for the ${ }^{4} C_{1 h 3 b}$ based structure. The full range of spin states has not been comprehensively investigated for all reaction paths since both previous calculations ${ }^{13}$ and a number of test calculations in this work demonstrate that, in general, spin multiplicity has a relatively small effect on the barrier heights and overall thermodynamics of reactions on TM clusters. However, we found two exceptions to this trend for reactions on the ${ }^{6} O_{4 h}$ structure (vide infra).

The reaction pathways have been calculated using eigenvector following ${ }^{41-46}$ to identify the reactant and product stationary points and, consequently, the barriers and overall thermodynamics of reaction. The zero-point energies of all of the minimum and TS structures along each of the paths were found to be very similar; therefore, the zero-point energy corrections do not alter the findings presented here. Consequently, the results are reported without zero-point corrections. A summary comprising the total energies of the reactant stationary points on the reaction pathways, the forward and reverse barriers and the energy difference between reactants and products are shown in Table III. The $O_{4 h}$ reactions are summarized for the quintet state (with two exceptions) and the $C_{1 h 3 b}$ reactions are summarized for the triplet state. While the $O_{4 h}$-TS1 reaction is found to be endothermic regardless of spin state, the reaction energetics show significant variation with spin, with the septet yielding the smaller reaction energy. Similarly, for $O_{4 h}$-TS5, the quintet pathway is barely endothermic, while the septet energetics yield an exothermic reaction.

Figure 6 shows the energetic profile of each path relative to the structure lowest in energy found in these pathway 


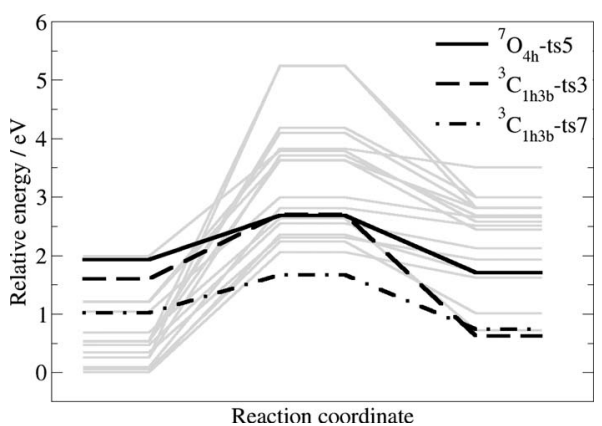

FIG. 6. Energetics for stationary points on reaction pathways for dissociation of nitric oxide on $\mathrm{Rh}_{6} \mathrm{O}_{4}^{+}$clusters. Reactants are on the left, TS at the center, and the dissociated products on the right. Reactant energies are relative to the reactant structure that is lowest in energy. The dark lines indicate the only reactions to be identified as exothermic.

calculations. Of all the reaction pathways to dissociation identified, only three are exothermic. Geometries of the stationary points along these three paths are shown in Fig. 7. The three exothermic paths all have high-energy reactant configurations, lying at least $1 \mathrm{eV}$ higher than the most favorable structure for adsorption on the given isomer. Also, for the ${ }^{7} \mathrm{O}_{4 h}$-TS5 pathway, the metal framework is also significantly distorted in the reactant structure where the NO is molecularly adsorbed. In addition, each of the three paths faces a significant barrier to reaction approximately $0.5-1$ $\mathrm{eV}$. The change in reactivity from exothermic to endothermic behavior for the $O_{4 h}$-TS5 pathway on changing from a septet to quintet multiplicity is due primarily to a change in the structure of the molecularly adsorbed reactant; in the septet the NO molecule is bound by both atoms while in the quintet a bridging, nitrogen-bound geometry is obtained. The majority of the pathways is endothermic and is not predicted to occur spontaneously. This suggests that the change in reactivity is due to NO molecules being unable to dissociate on the tetroxide cluster due to a combination of thermodynamic and kinetic control. Thermodynamic control arises from two factors; first, that all of the low-energy (high-abundance) reactants appear to support only endothermic reactions, and

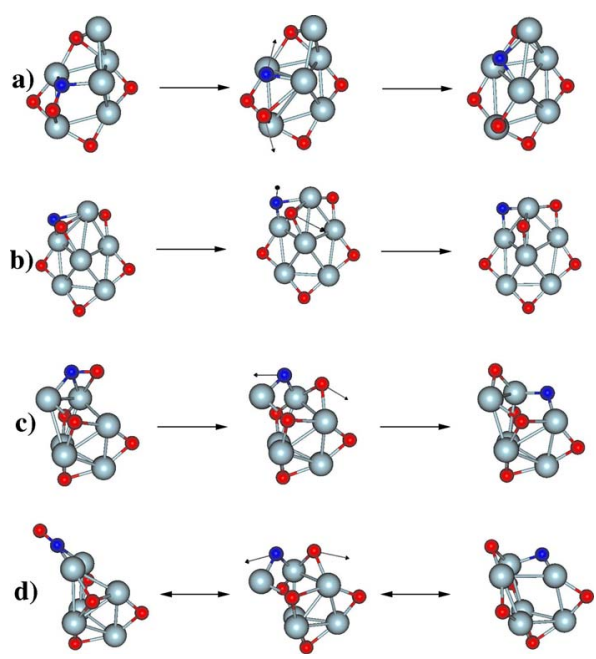

FIG. 7. (Color online) Geometries of stationary points on selected NO dissociation pathways on $\mathrm{Rh}_{6} \mathrm{O}_{4}^{+}$clusters: (a) ${ }^{3} C_{1 h 3 b}-\mathrm{TS} 3$, (b) ${ }^{3} C_{1 h 3 b}-\mathrm{TS} 7$, (c) ${ }^{7} \mathrm{O}_{4 h}$-TS5, and (d) ${ }^{5} \mathrm{O}_{4 h}$-TS5.

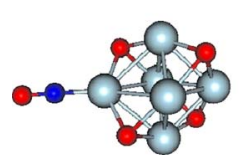

a)

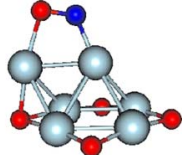

b)
FIG. 8. (Color online) Low energy structures for $\mathrm{Rh}_{6} \mathrm{O}_{4} \mathrm{NO}^{+}$clusters: (a) reactant from the ${ }^{5} \mathrm{O}_{4 h}$-TS3 pathway and (b) reactant from the ${ }^{3} C_{1 h 3 b}$-TS2 pathway.

second, those few reactant structures that do support exothermic reactions lie very high in energy relative to our predicted high-abundance structures. Further, the kinetic control arises from the predicted high barriers typically encountered for this reaction on the tetroxide clusters.

The lowest energy structures for molecular adsorption of NO on the ${ }^{6} O_{4 h}$ and ${ }^{4} C_{1 h 3 b}$ isomers, corresponding to the reactants mediated by the ${ }^{5} \mathrm{O}_{4 h}$-TS3 (and ${ }^{5} \mathrm{O}_{4 h}$-TS4) and ${ }^{3} C_{1 h 3 b}$-TS2 (and ${ }^{3} C_{1 h 3 b}$-TS4) pathways, are shown in Fig. 8. It can be seen that atop binding is favored on the octahedralbased cluster, while bridging via binding to both the nitrogen and oxygen atoms is favored on the csp-based cluster. These binding sites differ from those reported by Loffreda ${ }^{28,29}$ for the adsorption of NO on clean extended rhodium surfaces. It has not been our objective to make a thorough search for low-energy binding sites for molecularly adsorbed NO. Even so, our findings are consistent with the change in favored binding sites of oxygen atoms observed by us and by Mainardi and Balbuena ${ }^{17}$ between small clusters and bulk surfaces. These results strongly support the interpretation of the experimentally observed change in reactivity being due to the inability of the fifth, and subsequent, NO molecule(s) to dissociate upon adsorption, thereby preventing the formation and liberation of $\mathrm{N}_{2}$ from the cluster. Finally, to check if the presence of any oxygen atoms on the metal cluster would prevent dissociation of NO in our calculations, we have performed a similar search for reaction pathways on the $O_{2 h}$ dioxide isomer. In this instance we found a pathway that is exothermic by $0.5 \mathrm{eV}$, where the molecularly adsorbed reactant minimum supports an undistorted octahedral framework of rhodium atoms.

Clearly, the manifold of configuration and spin space that must be searched for the rhodium oxide clusters is large. Although it is credible that we have a reasonable estimate of the global minimum for each oxide composition, it is possible that there are other structures of lower energy that our search has not identified. Similar caveats apply to our search for TS, with the added complication that it is a more demanding task to amass evidence suggesting that a reaction is not likely to occur as opposed to finding a single example of an exothermic pathway. As highlighted in Sec. I, experimental confirmation of cluster structure is a challenging area. ${ }^{18,19}$ Plans to use far IR multiphoton dissociation spectroscopy on the rhodium oxide clusters in the near future are in place; our initial studies indicate the IR spectra of these structures will be sufficiently distinguishable in such experiments. A comparison of calculated and experimentally observed structures will be the subject of a future study and will be vital in validating our predictions and calibrating the DFT calculations. 


\section{CONCLUSIONS}

The structures of the oxide $\mathrm{Rh}_{6} \mathrm{O}_{m}^{+}$clusters $(1 \leq m \leq 4)$ have been investigated using DFT, and likely structural isomers have been identified. The relative abundance of isomers of $\mathrm{Rh}_{6} \mathrm{O}_{4}^{+}$has been calculated. The tetroxide structure of lowest energy was found to dominate the population only at very low total energies. The variation in favored spin multiplicity and the effects, in some cases, of changing spin multiplicity on the structures was found to significantly increase the complexity of searching the PES. The favored binding sites for oxygen atoms were found to depend on the cluster structure and the number of adsorbed oxygen atoms. Calculations of the energetics of reaction of nitric oxide on the two most abundant tetroxide clusters were found to be overwhelmingly endothermic. Three possible exothermic pathways found for this system were discounted due to high relative energy of the reactants and the high barriers encountered on these paths. The high energy of these anomalous reactants was attributed to the significant distortion of the metal atom framework. The location of an undistorted exothermic reaction pathway for the dioxide cluster further suggested that the coverage of four oxygen atoms, not the presence of oxygen atoms per se, appeared to hinder NO dissociation. The coverage of four oxygen atoms appears to be sufficient to block the remaining reaction sites available on the cluster.

\section{ACKNOWLEDGMENTS}

The authors gratefully acknowledge the computing facilities of the Centre for Scientific Computing, University of Warwick. D.J.H. is funded through a DTA award from the EPSRC. S.R.M. acknowledges the EPSRC for an Advanced Research Fellowship award.

${ }^{1}$ M. Knickelbein, Annu. Rev. Phys. Chem. 50, 79 (1999).

${ }^{2}$ A. Kaldor, D. M. Cox, D. J. Trevor, and M. R. Zakin, Z. Phys. D: At., Mol. Clusters 3, 195 (1986).

${ }^{3}$ M. B. Knickelbein and S. Yang, J. Chem. Phys. 93, 5760 (1990).

${ }^{4}$ B. A. Collings, D. M. Rayner, and P. A. Hackett, Int. J. Mass Spectrom. Ion Process. 125, 207 (1993).

${ }^{5}$ M. B. Knickelbein, Phys. Rev. A 67, 013202 (2003).

${ }^{6}$ A. J. Cox, J. G. Louderback, and L. A. Bloomfield, Phys. Rev. Lett. 71, 923 (1993).

${ }^{7}$ M. B. Knickelbein, J. Chem. Phys. 115, 1983 (2001).

${ }^{8}$ M. B. Knickelbein, J. Chem. Phys. 116, 9703 (2002).

${ }^{9}$ J. L. Elkind, F. D. Weiss, J. M. Alford, R. T. Laaksonen, and R. E. Smalley, J. Chem. Phys. 88, 5215 (1988).

${ }^{10}$ M. L. Anderson, M. S. Ford, P. J. Derrick, T. Drewello, D. P. Woodruff, and S. R. Mackenzie, J. Phys. Chem. A 110, 10992 (2006).
${ }^{11}$ D. Harding, M. S. Ford, T. R. Walsh, and S. R. Mackenzie, Phys. Chem. Chem. Phys. 9, 2130 (2007).

${ }^{12}$ C. Ratsch, A. Fielicke, A. Kirilyuk, J. Behler, G. von Helden, G. Meijer, and M. Scheffler, J. Chem. Phys. 122, 124302 (2005).

${ }^{13}$ D. Harding, S. R. Mackenzie, and T. R. Walsh, J. Phys. Chem. B 110, 18272 (2006).

${ }^{14}$ P. Armentrout, Annu. Rev. Phys. Chem. 52, 423 (2001).

${ }^{15}$ V. P. Zhdanov and B. Kasemo, Surf. Sci. Rep. 29, 31 (1997).

${ }^{16}$ M. S. Ford, M. L. Anderson, M. P. Barrow, D. P. Woodruff, T. Drewello, P. J. Derrick, and S. R. Mackenzie, Phys. Chem. Chem. Phys. 7, 975 (2005).

${ }^{17}$ D. S. Mainardi and P. B. Balbuena, J. Phys. Chem. A 107, 10370 (2003).

${ }^{18}$ A. Fielicke, G. v. Helden, G. Meijer, D. B. Pedersen, B. Simard, and D. M. Rayner, J. Phys. Chem. B 108, 14591 (2004).

${ }^{19}$ A. Fielicke, G. v. Helden, G. Meijer, D. Pedersen, B. Simard, and D. Rayner, J. Chem. Phys. 124, 194305 (2006).

${ }^{20}$ H. Grönbeck, A. Hellman, and A. Gavrin, J. Phys. Chem. A 111, 6062 (2007).

${ }^{21}$ G. L. Gutsev, M. D. Mochena, E. Johnson, and C. W. Bauschlicher, J. Chem. Phys. 125, 194312 (2006).

${ }^{22}$ S. Schwegmann, H. Over, V. D. Renzi, and G. Ertl, Surf. Sci. 375, 91 (1997).

${ }^{23}$ J. R. Mercer, P. Finetti, F. M. Leibsle, R. McGrath, V. R. Dhanak, A. Baraldi, K. C. Prince, and R. Rosei, Surf. Sci. 352-354, 173 (1996).

${ }^{24}$ D. Alfè, S. de Gironcoli, and S. Baroni, Surf. Sci. 410, 151 (1998).

${ }^{25}$ A. Baraldi, J. Cerdá, J. A. Martìn-Gago, G. Comelli, S. Lizzit, G. Paolucci, and R. Rosei, Phys. Rev. Lett. 82, 4874 (1999).

${ }^{26}$ A. G. Norris, F. Schedin, G. Thornton, V. R. Dhanak, T. S. Turner, and R. McGrath, Phys. Rev. B 62, 2113 (2000).

${ }^{27}$ J. E. Kirsch and S. Harris, Surf. Sci. 553, 82 (2004).

${ }^{28}$ D. Loffreda, F. Delbecq, D. Simon, and P. Sautet, J. Chem. Phys. 115, 8101 (2001).

${ }^{29}$ D. Loffreda, D. Simon, and P. Sautet, J. Catal. 213, 211 (2003).

${ }^{30}$ H. J. Borg, J. F. C.-J. M. Reijerse, R. A. van Santen, and J. W. Niemantsverdriet, J. Chem. Phys. 101, 10052 (1994).

${ }^{31}$ V. Stevanović, Z. Šljivančanin, and A. Baldereschi, Phys. Rev. Lett. 99, 165501 (2007).

${ }^{32}$ T. R. Walsh, J. Chem. Phys. 124, 204317 (2006).

${ }^{33}$ M. J. Frisch, G. W. Trucks, H. B. Schlegel et al., GAUssian, Gaussian, Inc., Pittsburgh, PA, 2003.

${ }^{34}$ J. P. Perdew, K. Burke, and M. Ernzerhof, Phys. Rev. Lett. 77, 3865 (1996).

${ }^{35}$ D. Andrae, U. Haussermann, M. Dolg, H. Stoll, and H. Preuss, Theor. Chim. Acta 77, 123 (1990).

${ }^{36}$ T. H. Dunning, Jr., J. Chem. Phys. 90, 1007 (1989).

${ }^{37}$ D. J. Wales, Mol. Phys. 78, 151 (1993).

${ }^{38}$ F. Raatz and D. R. Salahub, Surf. Sci. 146, L609 (1984).

${ }^{39}$ F. Raatz and D. R. Salahub, Surf. Sci. 176, 219 (1986).

${ }^{40}$ G. Pacchioni and P. Fantucci, Chem. Phys. Lett. 134, 407 (1987).

${ }^{41}$ J. Pancír, Collect. Czech. Chem. Commun. 40, 1112 (1975).

${ }^{42}$ J. Simons, P. Jørgenson, H. Taylor, and J. Ozment, J. Phys. Chem. 87, 2745 (1983).

${ }^{43}$ D. O'Neal, H. Taylor, and J. Simons, J. Phys. Chem. 88, 1510 (1984).

${ }^{44}$ A. Banerjee, N. P. Adams, J. Simons, and R. Shepard, J. Phys. Chem. 89, 52 (1985).

${ }^{45}$ J. Baker, J. Comput. Chem. 7, 385 (1986).

${ }^{46}$ J. Baker, J. Comput. Chem. 8, 563 (1987). 